\title{
Penetration and distribution of gadolinium-based contrast agents into the cerebrospinal fluid in healthy rats: a potential pathway of entry into the brain tissue
}

\author{
Gregor Jost $^{1} \cdot$ Thomas Frenzel $^{1} \cdot$ Jessica Lohrke $^{1} \cdot$ Diana Constanze Lenhard ${ }^{2}$. \\ Shinji Naganawa ${ }^{3} \cdot$ Hubertus Pietsch $^{1}$
}

Received: 14 September 2016 / Revised: 14 October 2016 / Accepted: 21 October 2016 / Published online: 10 November 2016

(C) The Author(s) 2016. This article is published with open access at Springerlink.com

\begin{abstract}
Objective Signal hyperintensity on unenhanced MRI in certain brain regions has been reported after multiple administrations of some, but not all, gadolinium-based contrast agents (GBCAs). One potential initial pathway of GBCA entry into the brain, infiltration from blood into the cerebrospinal fluid (CSF), was systematically evaluated in this preclinical study. Methods GBCA infiltration and distribution in the CSF were investigated in healthy rats using repeated fluid-attenuated MRI up to $4 \mathrm{~h}$ after high-dose $(1.8 \mathrm{mmol} / \mathrm{kg})$ administration of six marketed and one experimental GBCA. Additionally, gadolinium measurements in CSF, blood and brain tissue samples (after $24 \mathrm{~h}$ ) were performed using inductively coupled plasma mass spectrometry.

Results Enhanced MRI signals in the CSF spaces with similar distribution kinetics were observed for all GBCAs. No substantial differences in the gadolinium concentrations among the marketed GBCAs were found in the CSF, blood or brain tissue. After $4.5 \mathrm{~h}$, the concentration in the CSF was clearly higher than in blood but was almost completely cleared and lower than the brain tissue concentration after $24 \mathrm{~h}$.

Conclusions In contrast to the brain signal hyperintensities, no differences in penetration and distribution into the CSF of healthy rats exist among the marketed GBCAs.
\end{abstract}

Gregor Jost

gregor.jost@bayer.com

1 MR and CT Contrast Media Research, Bayer Pharma AG, Muellerstrasse 178, 13353 Berlin, Germany

2 Institute of Vegetative Physiology, Charité, Berlin, Germany

3 Department of Radiology, Nagoya University Graduate School of Medicine, Nagoya, Japan
Key Points

- Gadolinium-based contrast agents can cross the blood-CSF barrier.

- Fluid-attenuated MRI shows GBCA distribution with CSF flow.

- GBCA structure and physicochemical properties do not impact CSF penetration and distribution.

- GBCA clearance from CSF was almost complete within $24 \mathrm{~h}$ in rats.

- CSF is a potential pathway of GBCA entry into the brain.

Keywords Magnetic resonance imaging · Gadolinium • Contrast media $\cdot$ Cerebrospinal fluid $\cdot$ Brain

\section{Introduction}

Gadolinium-based contrast agents (GBCAs) are frequently used in MRI examinations and are generally considered to have an excellent safety profile [1]. Following intravenous injection, GBCAs distribute in the blood and the extravascular-extracellular space; however, to the common knowledge these agents cannot penetrate the intact bloodbrain barrier (BBB) [2]. Therefore, CNS imaging - i.e. the possibility to enhance areas with a disrupted $\mathrm{BBB}$ - is a major indication for contrast-enhanced MRI [3]. However, after multiple administrations of GBCAs, increased signal intensities (SIs) were recently reported on unenhanced T1-weighted MRIs in certain brain regions, mainly in patients with multiple sclerosis or neoplastic diseases [4-12]. A correlation between these signal hyperintensities in the dentate nucleus (DN) and globus pallidus (GP) and the number of contrast-enhanced MRI examinations was first described by Kanda et al. in 2014 [4]. Subsequent autopsy studies verified the presence of gadolinium in enhanced brain structures and suggest a 
correlation between the gadolinium present in the DN and GP and T1-weighted MRI SI increase [13, 14]. The SI increase seems to be primarily associated with the repeated use of the multi-purpose linear GBCAs gadodiamide, gadopentetate dimeglumine and gadobenate dimeglumine $[4-12,15,16]$. For gadopentetate dimeglumine a significant change of $\mathrm{T} 1$ was also confirmed by MR-relaxometry [17]. In the brain of decedents gadolinium was detected after administration of linear and macrocyclic GBCAs [18]. However, no visible SI increase has been observed for the macrocyclic GBCAs gadobutrol, gadoterate meglumine and gadoteridol [7, 11, 12, 19-21]. Stojanov et al. reported that gadobutrol causes signal enhancement of the DN and GP [22]. However, the design of this study was critically discussed and does not convincingly support this conclusion [23-25].

In order to study this more systematically and especially under controlled and reproducible conditions, animal models were established and confirmed prospectively the brain signal hyperintensities seen in the retrospective patient studies [26-28]. The animals showed elevated SIs in the cerebellar nuclei after multiple administrations of linear but not after receiving macrocyclic GBCAs [26-28]. It is important to note that the animals were healthy, without underlying diseases that may affect BBB integrity and function.

The mechanism of GBCA accumulation in the brain is unknown and raises several questions. This study focuses on the initial pathway of GBCA entry into the brain. In the brain, two barrier systems exist, the $\mathrm{BBB}$ and the blood-cerebrospinal fluid (CSF) barrier [29]. Regarding brain signal hyperintensities in clinical studies, the status of the patients' BBB integrity and function is either unknown or can be most likely characterized as a local $\mathrm{BBB}$ disruption due to disease processes. However, the underlying mechanism that allows GBCA infiltration into the brain, in particular in areas that are anatomically distant from a disease process, is not known. In addition to a disrupted BBB, GBCA penetration from the blood into the CSF represents another potential pathway for GBCA entry into the brain. Indeed, CSF enhancement in the internal auditory canal has been described after intravenous GBCA administration in patients with Ménière's disease [30, 31]. However, little knowledge exists about the impact of the physicochemical properties and chemical structure of GBCAs on the distribution and intensity of the enhancement. The impact of time on signal enhancement in various cranial fluid spaces after the administration of gadoteridol was evaluated in healthy volunteers using heavily T2-weighted fluid-attenuated inversion recovery (FLAIR) imaging [32]. This dedicated sequence is highly sensitive to detect low GBCA concentrations [30]. In a preclinical study, CSF signal enhancement on FLAIR images was initially observed for all GBCAs investigated, suggesting that all
GBCAs could pass the blood-CSF barrier in rats to a certain, but not yet quantified, extent [27].

The aim of this preclinical study was to evaluate the penetration of GBCAs from the blood into the CSF and the distribution kinetics within different CSF cavities in a systematic manner. Therefore, GBCAs with linear and macrocyclic structures and different physicochemical properties (ionic, nonionic and protein-binding) were evaluated by FLAIR MRI up to $4 \mathrm{~h}$ post injection (p.i.). In addition to the marketed GBCAs, the experimental macromolecular agent gadomer was used to assess the effect of molecular size. CSF and blood samples were obtained at 4.5 and $24 \mathrm{~h}$ after GBCA administration for inductively coupled plasma-mass spectrometry (ICP-MS)-based gadolinium quantification. Additionally, samples from the cerebellum and pons were analysed to determine gadolinium concentration 24 h p.i.

\section{Material and methods}

\section{Animals}

One hundred and two healthy Han-Wistar rats (Crl:WI; males; 275-325 g) were obtained from Charles River (Sulzfeld, Germany). The animals were kept under standard laboratory conditions and standard rat chow and water were provided ad libitum. The animals were handled and treated according to German animal regulations. The MRI and CSF sampling were performed under anaesthesia with $1.5 \%$ isoflurane (Baxter GmbH, Unterschleißheim, Germany).

\section{Study setup}

The study was performed in two parts: First, an MRI-based evaluation of GBCA infiltration and distribution within the CSF up to $4 \mathrm{~h}$ after the administration, and second, the quantification of CSF, blood, cerebellum and pons gadolinium concentration 24 h p.i. (Fig. 1). Ninety-six rats were randomly divided into a control and seven GBCA groups ( $\mathrm{n}=12$ each), and additional six rats were used to monitor quantitative changes of SI after a necessary service of the MRI system. For the two study parts, each group was divided into two subgroups $(\mathrm{n}=6$ each). The first subgroup underwent MRcisternography (MRC) and FLAIR MRI up to $4 \mathrm{~h}$ after GBCA administration. Subsequently, a CSF and blood sample was obtained for ICP-MS-based gadolinium quantification. Animals from the second subgroup underwent CSF and blood sampling $24 \mathrm{~h}$ after GBCA injection and samples from the cerebellum and pons were also taken. The following GBCAs were investigated: gadopentetate dimeglumine (Magnevist, Bayer Vital GmbH, Leverkusen, Germany), gadodiamide (Omniscan, GE Healthcare Buchler GmbH, Braunschweig, Germany), gadobenate dimeglumine (MultiHance, Bracco 


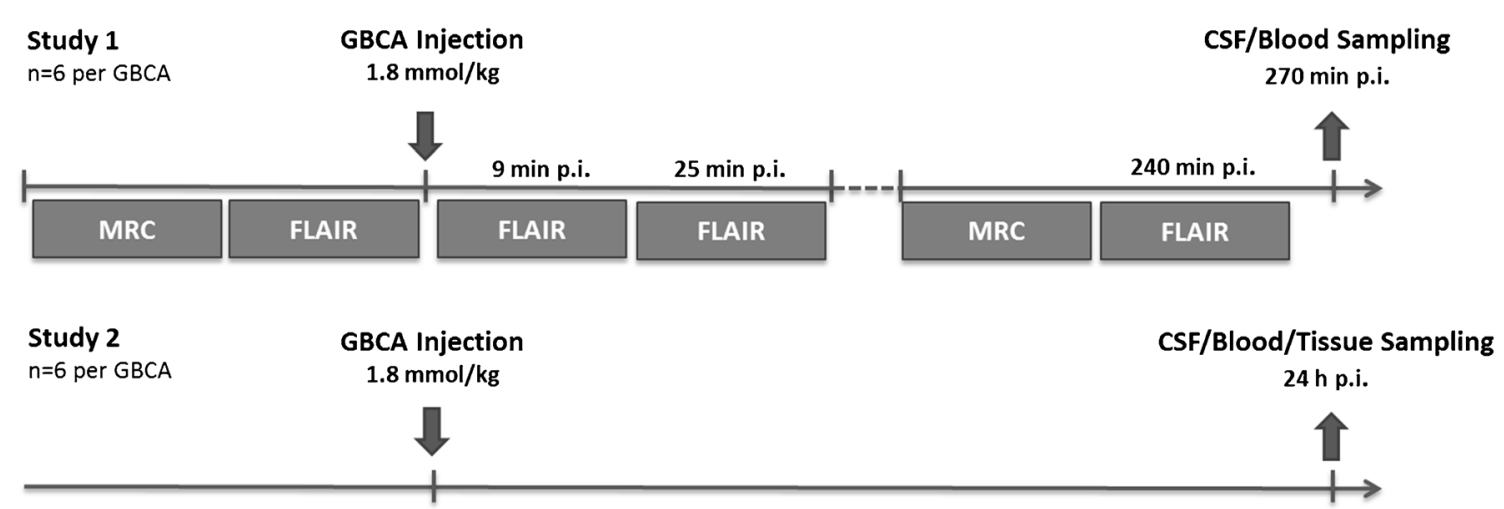

Fig. 1 Experimental setup. The study was performed in two parts, each with six animals per experimental group. Part 1 (upper row) included MR-cisternography (MRC) and fluid-attenuated imaging (FLAIR) of the rat brain before and up to $240 \mathrm{~min}$ after injection of GBCA. After
270 min a blood and CSF sampling was performed. In part 2 (lower row) blood, CSF and samples from the cerebellum and pons were obtained $24 \mathrm{~h}$ after injection. No MRI was done in this part of the study. p.i. post injection
Imaging Deutschland GmbH, Konstanz, Germany), gadobutrol (Gadovist, Bayer Vital $\mathrm{GmbH}$ ), gadoterate meglumine (Dotarem, Guerbet GmbH, Sulzbach/Taunus, Germany) and gadoteridol (ProHance, Bracco Imaging Deutschland $\mathrm{GmbH}$ ). The experimental GBCA gadomer (Bayer Pharma AG, Berlin, Germany) was used to assess the effect of molecular size. Gadomer is a dendritic gadolinium complex with a molecular weight of $17 \mathrm{kDa}$ that distributes almost exclusively within the intravascular compartment [33]. All agents were applied intravenously at a dose of $1.8 \mathrm{mmol} \mathrm{Gd} / \mathrm{kg}$ body weight. This dose approximates three times the human standard dose based upon body surface area normalization between rats and humans [34]. Saline administered at identical volume was used in the control group. During the experiments, servicing of the MR scanner was necessary. The images of the experimental gadomer and gadoteridol groups that underwent MRI after the service showed altered SI levels. Therefore, the additional gadobutrol group $(n=6)$ was added to ensure comparability between the first and second set of MRI measurements.

\section{MRI protocol}

MR imaging was performed using clinical 1.5-T MRI (Avanto, Siemens Healthcare GmbH, Erlangen, Germany) and a dedicated two-channel rat head coil (Rapid Biomedical GmbH, Rimpar, Germany). For the evaluation of the fluid space, T2-weighted MRC for anatomical references of the CSF space was used. For MRC, a variable flip angle 3D-TSE sequence $(\mathrm{TR}=4,400 \mathrm{~ms}, \mathrm{TE}=553 \mathrm{~ms}$ ) with an initial refocusing flip angle of $180^{\circ}$ (decreased to $120^{\circ}$ for the refocusing echo train) and a turbo factor of 79 were used. The spatial resolution was $0.3 \times 0.3 \times 0.6 \mathrm{~mm}$ (field of view $(\mathrm{FOV})=100 \times 48 \mathrm{~mm} ; 30$ transversal slices). MRC was followed by pre- and post-contrast heavily T2weighted FLAIR sequence with identical parameters.
However, a non-selective inversion recovery pulse with an inversion time of 2,250 ms was included, and TR was extended to 9,000 ms. The first FLAIR sequence was started $1 \mathrm{~min}$ after GBCA injection immediately followed by a second scan. The middle of the scan time (16:14 min) was set as the time point for the temporal evaluation ( $9 \mathrm{~min}$ and 25 min p.i., respectively). Another MRC and FLAIR measurement was performed $4 \mathrm{~h}$ p.i.

\section{Gadolinium quantification}

For CSF sampling, the anaesthetized animals were positioned in a stereotactic frame. The head was flexed, and a small incision was made inferior to the occiput to expose the dura mater of the cisterna magna. A catheter capillary tube was inserted into the cisterna magna, and the CSF was collected into the tube. The animals were euthanised by exsanguination, and a blood sample was collected. In the second part of the study, the brain was removed and dissected to sample the cerebellum and pons. The gadolinium concentrations of the CSF, blood, cerebellum and pons were measured by ICP-MS (Agilent 7500a, Waldbronn, Germany).

\section{Image evaluation}

Image analysis was performed by two experienced readers who were blinded to the experimental groups. In the MRC images, manually defined regions of interest (ROIs) were drawn in two adjacent slices around the third and fourth ventricles, the aqueduct and in the subarachnoid space at different anatomical locations (cerebral, spinal and lateral). Examples of the ROI placement are shown in Fig. 2. The ROI positions were copied to the FLAIR images to evaluate the contrast enhancement before and after GBCA administration. 


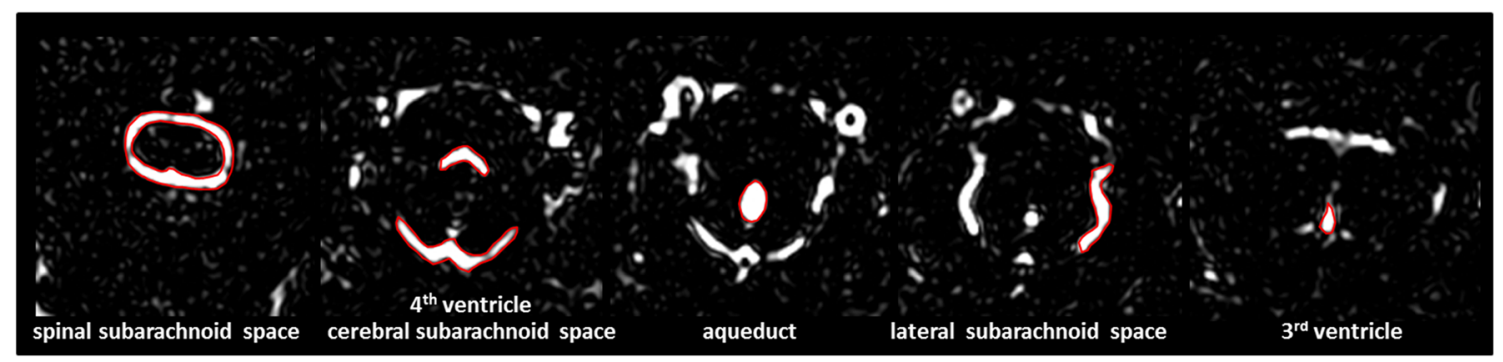

Fig. 2 Examples for region of interest (ROI) placement. For the quantitative analysis ROIs were placed around different CSF spaces using the MRcisternography (MRC) images for anatomical reference

\section{Results}

\section{CSF enhancement}

Signal enhancement of the CSF spaces was observed in all FLAIR images after GBCA administration. Figure 3 shows representative images of the brain at the level of the fourth ventricle. The cavity fluid spaces are visualized by MRC to determine the anatomical location and are almost completely attenuated in the baseline FLAIR scan (Fig. 3b). A clearly visible signal enhancement indicating the presence of GBCA in the fluid spaces was demonstrated by FLAIR imaging after $9 \mathrm{~min}, 25 \mathrm{~min}$ and $240 \mathrm{~min}$ p.i. (Fig. 3c-e). The FLAIR imaging throughout the brain depicted GBCA-induced signal enhancement in all ventricles and the subarachnoid space.

For quantitative image analysis ROIs were placed at different CSF spaces to evaluate the distribution pattern and kinetics of the GBCAs over 240 min (Fig. 4). Gadoteridol, gadomer and the second gadobutrol group were diagrammed separately (Fig. 4b) due to a shift in the SI levels after servicing of the MR scanner. Comparing the two gadobutrol groups (Fig. $4 \mathrm{a}$ and $b$ ), the different signal levels were obvious.

CSF signal enhancement was detected for all GBCAs at comparable levels. A rapid signal enhancement was found immediately after administration in the inner CSF cavities (third and fourth ventricle, aqueduct), which was followed by successively declining CSF signals. After $240 \mathrm{~min}$ the SI reached almost baseline level. By comparison, the CSF signal in the subarachnoid space (spinal cord, lateral and cerebral location) increased at a slower rate with a peak at 25 min p.i. Subsequently, decreasing SIs were observed until $240 \mathrm{~min}$ p.i., declining more rapidly and rigorously at the level of the spinal cord than on the lateral and cerebral level.

\section{Gadolinium quantification}

Analytical determinations of the gadolinium concentrations in the CSF and blood were conducted 4.5 and $24 \mathrm{~h}$ post GBCA injection by ICP-MS (Fig. 5). At $4.5 \mathrm{~h}$, the CSF gadolinium concentrations for the marketed GBCAs were in the range of $18.8 \pm 7.7 \mathrm{nmol} / \mathrm{ml}$ (gadobutrol) to $27.4 \pm 12.7 \mathrm{nmol} / \mathrm{ml}$ (gadoterate meglumine). For gadomer, a clearly lower concentration of $5.5 \pm 7.7 \mathrm{nmol} / \mathrm{ml} \mathrm{CSF}$ was detected. After $24 \mathrm{~h}$, the GBCAs were almost completely cleared from the CSF, and the concentrations of the marketed GBCAs ranged from 0.08 $\pm 0.02 \mathrm{nmol} / \mathrm{ml}$ (gadodiamide) to $0.28 \pm 0.16 \mathrm{nmol} / \mathrm{ml}$ (gadoterate meglumine). For gadomer, a gadolinium concentration of $0.07 \pm 0.02 \mathrm{nmol} / \mathrm{ml}$ was detected. Compared with blood, higher concentrations were found in the CSF for all marketed GBCAs at $4.5 \mathrm{~h}$. However, after $24 \mathrm{~h}$ all concentrations in the CSF were below the blood gadolinium levels. In the blood, comparable gadolinium concentrations were observed $4.5 \mathrm{~h}$ after administration of the marketed GBCAs. The concentration ranged from $2.0 \pm 0.7 \mathrm{nmol} / \mathrm{ml}$ (gadobenate dimeglumine) to $4.7 \pm 2.4 \mathrm{nmol} / \mathrm{ml}$ (gadodiamide). After $24 \mathrm{~h}$, the remaining concentrations were in the range between 0.27

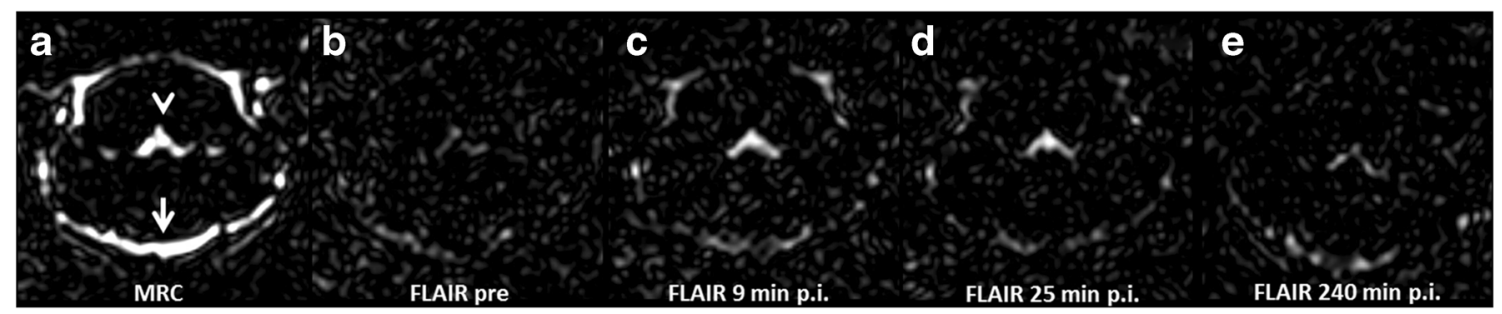

Fig. 3 Representative images. The CSF spaces were visualised by MRcisternography (MRC), for example the fourth ventricle (arrowhead) and the subarachnoid space (arrow) (a). In the fluid-attenuated (FLAIR) images before GBCA injection the respective CSF signal is almost completely attenuated (b). After GBCA administration a clear signal enhancement of the CSF spaces was found in the FLAIR images up to 240 min post injection (p.i.) (c-e) 

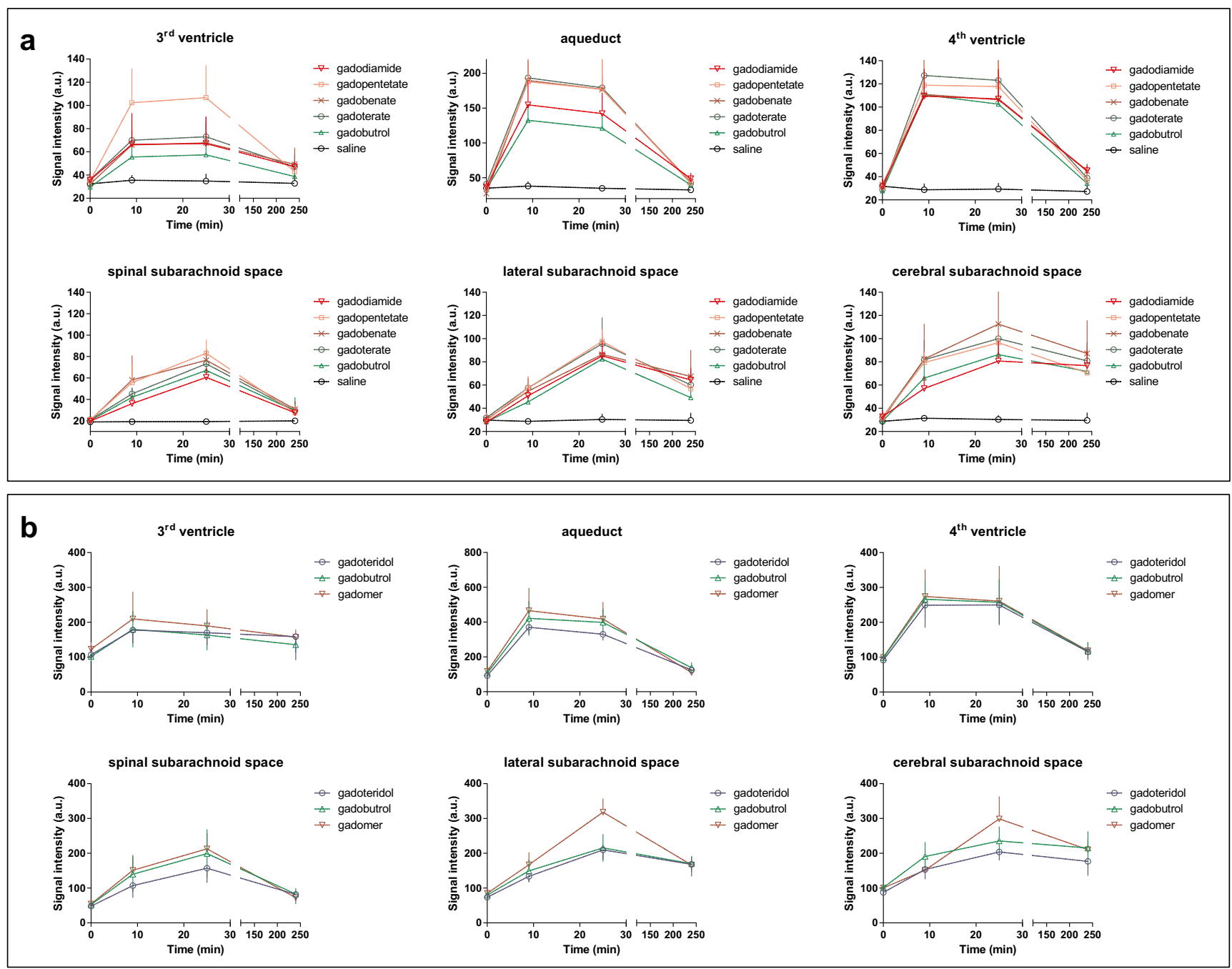

Fig. 4 Quantitative analysis of CSF signal intensity (SI). The analysis of the SI on fluid-attenuated (FLAIR) images over time in different CSF spaces was divided into two parts ( $\mathbf{a}$ and $\mathbf{b}$ ) because the SI for the baseline scans $(t=0 \mathrm{~min})$ was significantly higher after servicing of the
MRI scanner. However, each analysis includes a gadobutrol group to ensure comparability. gadopentetate $=$ gadopentetate dimeglumine; gadobenate $=$ gadobenate dimeglumine; gadoterate $=$ gadoterate meglumine; error bars represent standard deviation
Fig. 5 Gadolinium (Gd) concentrations in CSF and blood. The gadolinium concentration determined in the CSF (b) and blood (b) samples obtained at $4.5 \mathrm{~h}$ and $24 \mathrm{~h}$, respectively. gadopentetate $=$ gadopentetate dimeglumine; gadobenate $=$ gadobenate dimeglumine; gadoterate $=$ gadoterate meglumine; error bars represent standard deviation

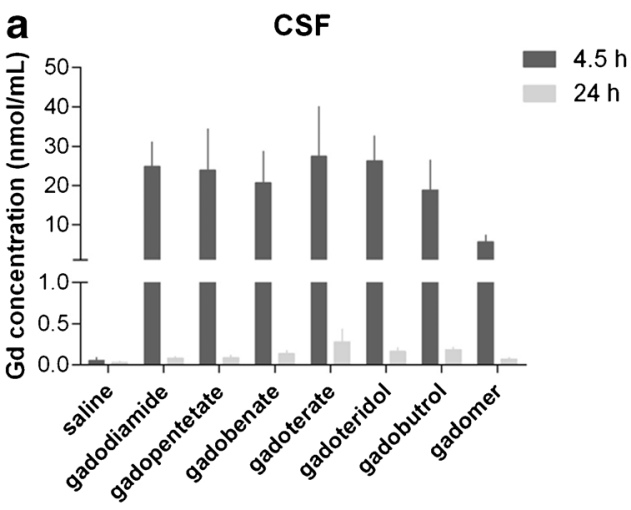

b

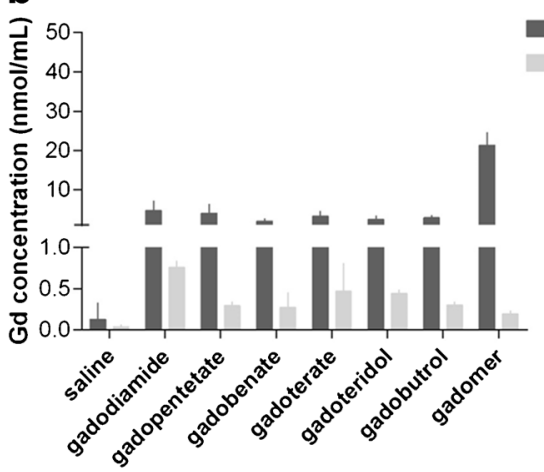

$4.5 \mathrm{~h}$

$24 \mathrm{~h}$ 
$\pm 0.19 \mathrm{nmol} / \mathrm{ml}$ (gadobenate dimeglumine) and 0.76 $\pm 0.08 \mathrm{nmol} / \mathrm{ml}$ (gadodiamide).

The gadolinium concentrations measured in the cerebellum and pons obtained $24 \mathrm{~h}$ p.i. were higher than those in the CSF and blood. In the cerebellum, the concentrations for the marketed GBCAs were in the range of $0.63 \pm 0.05 \mathrm{nmol} / \mathrm{g}$ (gadoteridol) to $1.06 \pm 0.05 \mathrm{nmol} / \mathrm{g}$ (gadodiamide). In the pons, slightly lower gadolinium concentrations were detected. In both structures analysed, the lowest concentrations were found for gadomer (Fig. 6).

\section{Discussion}

In this preclinical rat study, the amount and kinetics of GBCA infiltration and distribution in the CSF were investigated by FLAIR MRI up to $4 \mathrm{~h}$ p.i. Additionally, gadolinium measurements in the CSF, blood cerebellum and pons were performed by ICP-MS. For this purpose, six marketed GBCAs with different structural and physicochemical properties and one experimental agent with significantly larger molecular size were evaluated.

Comparable CSF signal enhancements on FLAIR images were observed for all GBCAs independent of their chemical structure or physicochemical properties such as the ionicity or ability to bind partially to proteins. The kinetics of signal enhancement differs between the inner CSF cavities (ventricles and aqueduct) and subarachnoid space. The faster signal increase in the inner cavities demonstrates that the primary location of GBCA infiltration is most likely the choroid plexus located in the ventricles. The fenestrated capillaries of the choroid plexus are relatively permeable to smaller substances, such as GBCAs, which can pass into the choroid plexus interstitium. The choroid plexus continuously secretes CSF, and the choroid plexus epithelium forms the blood-CSF barrier.
Importantly, the blood-CSF barrier is known to be physiologically more leaky than the BBB $[3,35]$.

This study demonstrated that all marketed GBCAs cross the blood-CSF barrier to an almost identical extent. However, the ability to cross this barrier seems to depend on the molecular size as demonstrated by the considerably lower CSF gadolinium concentration for the experimental gadomer which is significantly larger $(17 \mathrm{kDa})$ than the marketed GBCAs $(<1 \mathrm{kDa})$. In contrast to the analytical gadolinium quantification, the reduction was not observed with FLAIR MRI as the r1 relaxivity of gadomer is about a factor of five higher than that of the other GBCAs [36]. After penetrating the blood-CSF barrier, further GBCA distribution within the CSF is driven by diffusion, convection and CSF flow that are directed through the ventricles to the subarachnoid space of the cortex and spinal cord. The delayed MRI signal increase observed in the subarachnoid space represents this distribution process.

For the marketed GBCAs, the averaged CSF gadolinium concentrations are about a factor of 7.4 higher than the respective blood concentrations at $4.5 \mathrm{~h}$ p.i. However, after $24 \mathrm{~h}$ GBCAs are almost completely cleared from the CSF, and the respective gadolinium concentrations are much lower than those in the blood. This is in contrast to gadolinium concentrations in the cerebellum and pons that are higher than those in the CSF and blood at $24 \mathrm{~h}$ p.i. This demonstrates that all GBCAs can be found in the brains of rats $24 \mathrm{~h}$ after the administration. Slight quantitative differences between the agents seem to exist. In the cerebellum of animals administered gadodiamide, the gadolinium concentration was higher than that of the other GBCAs (Fig. 6).

GBCA kinetics of distribution and excretion from the cerebellum and pons at later time points cannot be described with this study. However, in a recently published mouse study, the brain gadolinium concentration decreased between 3 and 45 days p.i., indicating a persistent GBCA elimination from
Fig. 6 Gadolinium (Gd) concentrations in the cerebellum and pons. The gadolinium concentration per gram tissue determined in the cerebellum (a) and pons (b) $24 \mathrm{~h}$ after administration. gadopentetate $=$ gadopentetate dimeglumine; gadobenate $=$ gadobenate dimeglumine; gadoterate $=$ gadoterate meglumine; error bars represent standard deviation
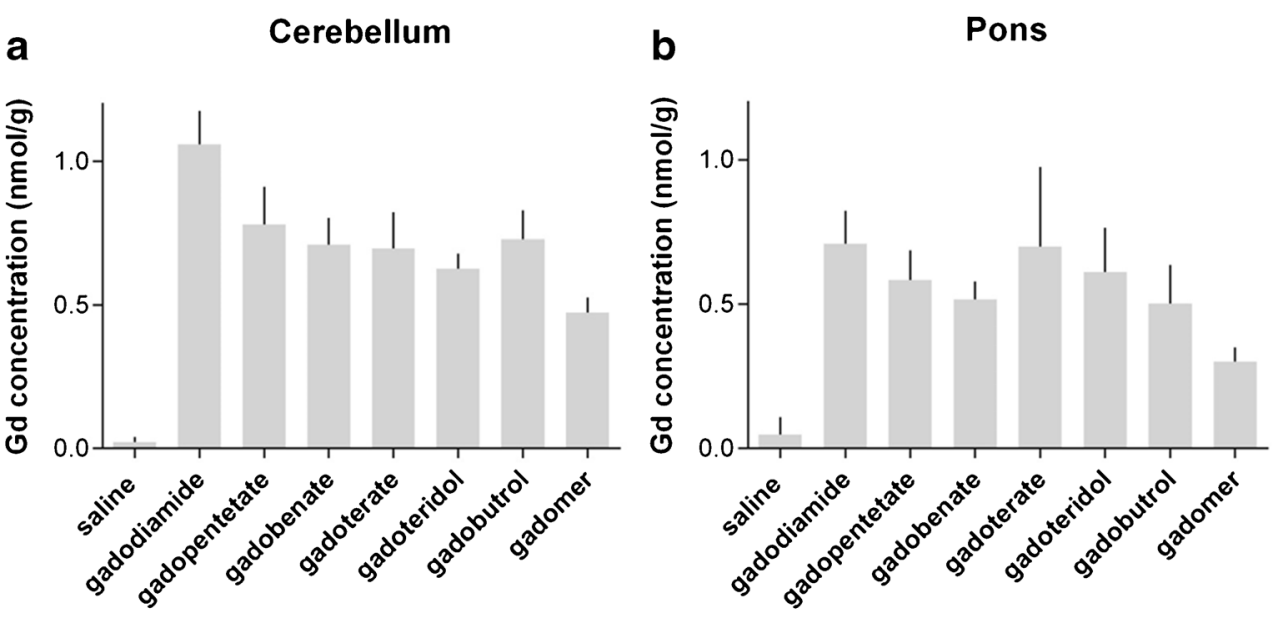
the brain tissue during this period [37]. Clinical studies have also demonstrated clear differences in brain signal hyperintensity between GBCAs that are associated with their chemical structure $[7,11,12]$. This was confirmed in preclinical investigations by ICP-MS-based brain gadolinium measurements 5 weeks p.i. $[26,28]$. The finding of our study, i.e. the fact that all GBCAs independently of their chemical structure initially reach the cerebellum and pons, leads to the hypothesis that the GBCA complex stability plays a role during further elimination from this brain structures. The different chemical structures exhibit different thermodynamic and kinetic complex stabilities [38, 39], apparently allowing for greater release of $\mathrm{Gd}^{3+}$ at physiological conditions with linear than with macrocyclic GBCAs [40]. However, to assess the role of complex stability, the chemical species of gadolinium in the brain has to be evaluated. ICP-MS can quantify the gadolinium concentration but cannot distinguish between different forms of gadolinium (e.g. chelated or bound to other chemical species). The development of advanced analytical methods for gadolinium speciation would lead to a more detailed understanding of gadolinium retention in the brain and would shed more light on the role of complex stability.

Although we demonstrated the infiltration and distribution of GBCAs into the CSF, the further GBCA distribution into the brain parenchyma is not conclusive. Assuming that the GBCA in the CSF represents the source of the gadolinium found in the cerebellum and pons, different pathways of distribution might exist. In the classical view, the penetration of substances from the CSF into the brain parenchyma is mediated by diffusion, a process that is much slower than CSF convection and flow. Hence, this process is not very effective and restricted to the upper surface of the brain parenchyma [41]. The perivascular fluid circulation through the VirchowRobin space surrounding the pial arteries might represent a more efficient route of GBCA transport from the CSF to the brain parenchyma. Recent studies have demonstrated that CSF and interstitial fluid continuously interchange [42]. It is likely that the underlying pathway termed glymphatic system plays an important role in GBCA infiltration from the CSF into the brain tissue [43]. An enhancement of the perivascular space was recently confirmed in humans $4 \mathrm{~h}$ after administration of gadoteridol or gadodiamide [44]. However, to date, no connection can be made between the GBCA found in the CSF and the accumulation of gadolinium in the brain.

This study has certain limitations. It was performed on healthy rats without known BBB disorders. This can approach but cannot fully replicate the clinical conditions in patients, especially regarding BBB integrity and function. Gadolinium was quantified in the cerebellum and pons; however, no histological evaluation of these structures was performed. Other limitations arose from the used MRI method. The altered SI levels after MRI scanner servicing do not allow a direct comparison of the two subgroups. In addition, the SI of the heavily
T2-weighted FLAIR sequence depends on the T1 and T2 relaxation times of the CSF in a complex manner. Since GBCAs possess different $\mathrm{r} 1$ and $\mathrm{r} 2$ relaxivites in water [36], a correlation between SI and GBCA concentration is not a constant factor and differs among agents. Thus, FLAIR imaging is more sensitive for GBCAs with a high r1 (e.g. gadobenate dimeglumine) than for GBCA with a lower $\mathrm{r} 1$ (e.g. gadoterate meglumine and gadoteridol) [36]. However, gadolinium concentration measurements in the CSF $4.5 \mathrm{~h}$ p.i. generally confirm the MRI results.

In summary, this study shows that GBCAs can penetrate from blood into the CSF independent of their chemical structure or physicochemical properties. Only the molecular size seems to be an important parameter as demonstrated by a lower CSF gadolinium concentration after administration of the macromolecular agent gadomer. Dynamic FLAIR MRI demonstrates a kinetic from the inner CSF spaces to the subarachnoid space and suggests a passive distribution and washout driven by convection and CSF flow. Twenty-four hours after injection, GBCA clearance from the CSF was almost complete, whereas slightly higher gadolinium concentrations were found in the cerebellum and pons, suggesting delayed excretion from these structures. To date, the mechanism of final distribution from the CSF into the brain and specifically to the DN and the GP could not be evaluated in this experiment and needs further study.

Acknowledgments The scientific guarantor of this publication is $\mathrm{H}$. Pietsch. The authors of this manuscript declare relationships with the following companies: G. Jost, T. Frenzel, J. Lohrke and H. Pietsch are employees of Bayer Pharma AG. D.C. Lenhard received a research grant from Bayer Pharma AG. No complex statistical methods were necessary for this paper. Methodology: prospective, experimental, performed at one institution

Open Access This article is distributed under the terms of the Creative Commons Attribution 4.0 International License (http:// creativecommons.org/licenses/by/4.0/), which permits unrestricted use, distribution, and reproduction in any medium, provided you give appropriate credit to the original author(s) and the source, provide a link to the Creative Commons license, and indicate if changes were made.

\section{References}

1. Matsumura T, Hayakawa M, Shimada F et al (2013) Safety of gadopentetate dimeglumine after 120 million administrations over 25 years of clinical use. Magn Reson Med Sci 12:297-304

2. Weinmann HJ, Brasch RC, Press WR, Wesbey GE (1984) Characteristics of gadolinium-DTPA complex: a potential NMR contrast agent. AJR Am J Roentgenol 142:619-624

3. Sage MR, Wilson AJ (1994) The blood-brain barrier: an important concept in neuroimaging. AJNR Am J Neuroradiol 15:601-622

4. Kanda T, Ishii K, Kawaguchi H, Kitajima K, Takenaka D (2014) High signal intensity in the dentate nucleus and globus pallidus on unenhanced T1-weighted MR images: relationship with increasing cumulative dose of a gadolinium-based contrast material. Radiology 270:834-841 
5. Errante Y, Cirimele V, Mallio CA, Di Lazzaro V, Zobel BB, Quattrocchi CC (2014) Progressive increase of T1 signal intensity of the dentate nucleus on unenhanced magnetic resonance images is associated with cumulative doses of intravenously administered gadodiamide in patients with normal renal function, suggesting dechelation. Investig Radiol 49:685-690

6. Quattrocchi CC, Mallio CA, Errante Y et al (2015) Gadodiamide and dentate nucleus $\mathrm{T} 1$ hyperintensity in patients with meningioma evaluated by multiple follow-up contrast-enhanced magnetic resonance examinations with no systemic interval therapy. Investig Radiol 50:470-472

7. Kanda T, Osawa M, Oba $\mathrm{H}$ et al (2015) High signal intensity in dentate nucleus on unenhanced T1-weighted MR images: association with linear versus macrocyclic gadolinium chelate administration. Radiology 275:803-809

8. Ramalho J, Castillo M, AlObaidy M et al (2015) High signal intensity in globus pallidus and dentate nucleus on unenhanced T1weighted MR images: evaluation of two linear gadolinium-based contrast agents. Radiology 276:836-844

9. Adin ME, Kleinberg L, Vaidya D, Zan E, Mirbagheri S, Yousem DM (2015) Hyperintense dentate nuclei on T1-weighted MRI: relation to repeat gadolinium administration. AJNR Am J Neuroradiol 36:1859-1865

10. Weberling LD, Kieslich PJ, Kickingereder P et al (2015) Increased signal intensity in the dentate nucleus on unenhanced T1-weighted images after gadobenate dimeglumine administration. Investig Radiol 50:743-748

11. Radbruch A, Weberling LD, Kieslich PJ et al (2015) Gadolinium retention in the dentate nucleus and globus pallidus is dependent on the class of contrast agent. Radiology 275:783-791

12. Cao Y, Huang DQ, Shih G, Prince MR (2016) Signal change in the dentate nucleus on T1-weighted MR images after multiple administrations of gadopentetate dimeglumine versus gadobutrol. AJR Am J Roentgenol 206:414-419

13. McDonald RJ, McDonald JS, Kallmes DF et al (2015) Intracranial gadolinium deposition after contrast-enhanced MR imaging. Radiology 275:772-782

14. Kanda T, Fukusato T, Matsuda M et al (2015) Gadolinium-based contrast agent accumulates in the brain even in subjects without severe renal dysfunction: evaluation of autopsy brain specimens with inductively coupled plasma mass spectroscopy. Radiology 276:228-232

15. Ramalho J, Semelka RC, AlObaidy M, Ramalho M, Nunes RH, Castillo M (2016) Signal intensity change on unenhanced T1weighted images in dentate nucleus following gadobenate dimeglumine in patients with and without previous multiple administrations of gadodiamide. Eur Radiol

16. Zhang Y, Cao Y, Shih GL, Hecht EM, Prince MR (2016) Extent of signal hyperintensity on unenhanced T1-weighted brain MR images after more than 35 administrations of linear gadoliniumbased contrast agents. Radiology: 152864

17. Tedeschi E, Palma G, Canna A, et al. (2016) In vivo dentate nucleus MRI relaxometry correlates with previous administration of Gadolinium-based contrast agents. Eur Radiol

18. Murata N, Gonzalez-Cuyar LF, Murata K et al (2016) Macrocyclic and other non-group 1 gadolinium contrast agents deposit low levels of gadolinium in brain and bone tissue: preliminary results from 9 patients with normal renal function. Investig Radiol 51:447-453

19. Radbruch A, Weberling LD, Kieslich PJ et al (2015) High-signal intensity in the dentate nucleus and globus pallidus on unenhanced T1-weighted images: evaluation of the macrocyclic gadoliniumbased contrast agent gadobutrol. Investig Radiol 50:805-810

20. Kromrey ML, Liedtke KR, Ittermann T, et al. (2016) Erratum to: Intravenous injection of gadobutrol in an epidemiological study group did not lead to a difference in relative signal intensities of certain brain structures after 5 years. Eur Radiol
21. Kromrey ML, Liedtke KR, Ittermann T, et al. (2016) Intravenous injection of gadobutrol in an epidemiological study group did not lead to a difference in relative signal intensities of certain brain structures after 5 years. Eur Radiol

22. Stojanov DA, Aracki-Trenkic A, Vojinovic S, BenedetoStojanov D, Ljubisavljevic S (2015) Increasing signal intensity within the dentate nucleus and globus pallidus on unenhanced $\mathrm{T} 1 \mathrm{~W}$ magnetic resonance images in patients with relapsingremitting multiple sclerosis: correlation with cumulative dose of a macrocyclic gadolinium-based contrast agent, gadobutrol. Eur Radiol 26:807-815

23. Agris J, Pietsch H, Balzer T (2015) What evidence is there that gadobutrol causes increasing signal intensity within the dentate nucleus and globus pallidus on unenhanced T1W MRI in patients with RRMS? Eur Radiol 26:816-817

24. Stojanov DA (2015) Reply to Letter to the Editor re: Increasing signal intensity within the dentate nucleus and globus pallidus on unenhanced $\mathrm{T} 1 \mathrm{~W}$ magnetic resonance images in patients with relapsing-remitting multiple sclerosis: Correlation with cumulative dose of a macrocyclic gadolinium-based contrast agent, gadobutrol. Eur Radiol 26:816-817

25. Runge VM (2015) Macrocyclic versus linear gadolinium chelates. Investig Radiol 50:811

26. Robert P, Lehericy S, Grand S et al (2015) T1-weighted hypersignal in the deep cerebellar nuclei after repeated administrations of gadolinium-based contrast agents in healthy rats: difference between linear and macrocyclic agents. Investig Radiol 50:473-480

27. Jost G, Lenhard DC, Sieber MA, Lohrke J, Frenzel T, Pietsch H (2016) Signal increase on unenhanced T1-weighted images in the rat brain after repeated, extended doses of gadolinium-based contrast agents: comparison of linear and macrocyclic agents. Investig Radiol 51:83-89

28. Robert P, Violas X, Grand S et al (2016) Linear gadolinium-based contrast agents are associated with brain gadolinium retention in healthy rats. Investig Radiol 51:73-82

29. Engelhardt B, Sorokin L (2009) The blood-brain and the bloodcerebrospinal fluid barriers: function and dysfunction. Semin Immunopathol 31:497-511

30. Naganawa S, Kawai H, Sone M, Nakashima T (2010) Increased sensitivity to low concentration gadolinium contrast by optimized heavily T2-weighted 3D-FLAIR to visualize endolymphatic space. Magn Reson Med Sci 9:73-80

31. Naganawa S, Nakashima T (2014) Visualization of endolymphatic hydrops with MR imaging in patients with Meniere's disease and related pathologies: current status of its methods and clinical significance. Jpn J Radiol 32:191-204

32. Naganawa S, Suzuki K, Yamazaki M, Sakurai Y (2014) Serial scans in healthy volunteers following intravenous administration of gadoteridol: time course of contrast enhancement in various cranial fluid spaces. Magn Reson Med Sci 13:7-13

33. Misselwitz B, Schmitt-Willich H, Ebert W, Frenzel T, Weinmann HJ (2001) Pharmacokinetics of Gadomer-17, a new dendritic magnetic resonance contrast agent. MAGMA 12:128-134

34. U.S. Department of Health and Human Devices FaDA Center for Drug Evaluation and Research (2005) Estimating the maximum safe starting dose in clinical trials for therapeutics in adult healthy volunteers

35. Pardridge WM (2011) Drug transport in brain via the cerebrospinal fluid. Fluids Barriers CNS 8:7

36. Rohrer M, Bauer H, Mintorovitch J, Requardt M, Weinmann HJ (2005) Comparison of magnetic properties of MRI contrast media solutions at different magnetic field strengths. Investig Radiol 40:715-724

37. Kartamihardja AA, Nakajima T, Kameo S, Koyama H, Tsushima Y (2016) Impact of impaired renal function on gadolinium retention 
after administration of gadolinium-based contrast agents in a mouse model. Investig Radiol

38. Port M, Idee JM, Medina C, Robic C, Sabatou M, Corot C (2008) Efficiency, thermodynamic and kinetic stability of marketed gadolinium chelates and their possible clinical consequences: a critical review. Biometals 21:469-490

39. Idee JM, Port M, Robic C, Medina C, Sabatou M, Corot C (2009) Role of thermodynamic and kinetic parameters in gadolinium chelate stability. J Magn Reson Imaging 30:1249-1258

40. Frenzel T, Lengsfeld P, Schirmer H, Hutter J, Weinmann HJ (2008) Stability of gadolinium-based magnetic resonance imaging contrast agents in human serum at 37 degrees C. Investig Radiol 43:817-828
41. Blasberg RG, Patlak C, Fenstermacher JD (1975) Intrathecal chemotherapy: brain tissue profiles after ventriculocisternal perfusion. J Pharmacol Exp Ther 195:73-83

42. Iliff JJ, Wang M, Liao Y et al (2012) A paravascular pathway facilitates CSF flow through the brain parenchyma and the clearance of interstitial solutes, including amyloid beta. Sci Transl Med 4:147ra111

43. Jessen NA, Munk AS, Lundgaard I, Nedergaard M (2015) The glymphatic system: a beginner's guide. Neurochem Res 40:2583-2599

44. Naganawa S, Nakane T, Kawai H, Taoka T (2016) Gd-based contrast enhancement of the perivascular spaces in the basal ganglia. Magn Reson Med Sci 